\title{
Genesis of a novel Shigella flexneri serotype by sequential infection of serotype-converting bacteriophages $\mathrm{SfX}$ and $\mathrm{Sfl}$
}

Qiangzheng Sun ${ }^{1 \dagger}$, Ruiting Lan ${ }^{2 \dagger}$, Yiting Wang ${ }^{1 \dagger}$, Jianping Wang ${ }^{1 \dagger}$, Xia Luo ${ }^{1 \dagger}$, Shaomin Zhang ${ }^{1}$, Peijing Li ${ }^{1}$, Yan Wang ${ }^{1}$, Changyun Ye ${ }^{1}$, Huaiqi Jing ${ }^{1}$ and Jianguo $\mathrm{Xu}^{1 *}$

\section{Clinical microbiology and vaccines}

\begin{abstract}
Background: Shigella flexneri is the major pathogen causing bacillary dysentery. Fifteen serotypes have been recognized up to now. The genesis of new S. flexneri serotypes is commonly mediated by serotype-converting bacteriophages. Untypeable or novel serotypes from natural infections had been reported worldwide but have not been generated in laboratory.

Results: A new S. flexneri serotype-serotype $1 \mathrm{~d}$ was generated when a S. flexneri serotype Y strain (native LPS) was sequentially infected with 2 serotype-converting bacteriophages, $\mathrm{SfX}$ first and then $\mathrm{Sfl}$. The new serotype $1 \mathrm{~d}$ strain agglutinated with both serotype $\mathrm{X}$-specific anti-7;8 grouping serum and serotype 1a-specific anti- I typing serum, and differed from subserotypes $1 \mathrm{a}, 1 \mathrm{~b}$ and $1 \mathrm{c}$. Twenty four $\mathrm{S}$. flexneri clinical isolates of serotype $\mathrm{X}$ were all converted to serotype $1 \mathrm{~d}$ by infection with phage Sfl. PCR and sequencing revealed that $\mathrm{Sfl}$ and SfX were integrated in tandem into the proA-yaiC region of the host chromosome.

Conclusions: These findings suggest a new S. flexneri serotype could be created in nature. Such a conversion may be constrained by susceptibility of a strain to infection by a given serotype-converting bacteriophage. This finding has significant implications in the emergence of new S. flexneri serotypes in nature.
\end{abstract}

\section{Background}

Shigella is the primary pathogen causing bacillary dysentery in developing countries. There are an estimated 164.7 million people worldwide infected by Shigella annually; resulting in 1.1 million deaths, most being children under five years [1]. A more recent study estimated approximately 125 million annual shigellosis cases and 14,000 related deaths in Asia [2], suggesting that the death rate has decreased significantly in recent years. Among the four Shigella species, S. dysenteriae, S.

\footnotetext{
* Correspondence: xujianguo@icdc.cn

† Contributed equally

'State Key Laboratory for Infectious Disease Prevention and Control, National Institute for Communicable Disease Control and Prevention, China CDC, P.O. Box 5, Changping, Beijing, China

Full list of author information is available at the end of the article
}

flexneri, S. boydii, and S. sonnei, S. flexneri is the predominant species [3].

S. flexneri serotyping are based on structure of the Oantigen lipopolysaccharide. There are 15 known serotypes: 1a, 1b, 1c, 2a, 2b, 3a, 3b, 4a, 4b, 5a, 5b, 6, X, Xv and $Y[4,5]$. Except for serotype 6, all share a common tetrasaccharide backbone of repeating units of $\mathrm{N}$-acetylglucosamine-rhamnose-rhamnose-rhamnose [6]. By adding glucosyl and/or $O$-acetyl groups to one or more of the sugars on the tetrasaccharide unit, various serotypes are formed. Serotype $\mathrm{Y}$ possesses the primary basic Oantigen without any modification of the tetrasaccharide backbone [6].

It is well known that $S$. flexneri serotype conversion is mediated by temperate bacteriophages [6,7]. Six different serotype-converting phages or prophages, SfI, SfII, Sf6,

\section{Biomed Central}


SfIV, SfV and SfX, have been identified and characterized [8-12], which can convert serotype $Y$ to serotype 1a, 2a, 3b, 4a, 5a and X respectively [8-12]. Except for Sf6 which carries a single gene, oac, for acetylation of the O-antigen [13], the other phages carry three genes, $g \operatorname{tr} A, g \operatorname{tr} B$, and $g \operatorname{tr}_{\text {type }}$ for O-antigen modification. The first two gtr genes are highly conserved and interchangeable in function, while the third gtr gene encodes a typespecific glucosyltransferase responsible for the addition of glucosyl molecules to sugar residue(s) on the basic $\mathrm{O}$-antigen repeating unit $[9,12,14]$. These phages integrate into the $S$. flexneri host chromosome either at tRNA-thrW downstream of proA [15] or at tRNA-argW adjacent to $y f d C$ [11]. Once integrated, the int and Oantigen modification genes are located at the opposition ends of the prophage genome, flanked by an attL sequence on the left and an $a t t R$ sequence on the right [15].

Recently, untypeable or novel serotypes of S. flexneri from natural infections had been reported worldwide $[5,16,17]$. A novel serotype 1c was identified in Bangladesh in the late 1980s and was a predominant serotype in Vietnam and other Asian countries [16,17]. Serotype $1 \mathrm{c}$ was a result of modification of serotype $1 \mathrm{a}$ with addition of a glucosyl group by a cryptic prophage carrying a gtr1C gene cluster [18]. More recently, a new serotype named as Xv emerged in China, and replaced 2a to become the most prevalent $S$. flexneri serotype [5]. Although the antigenic determinant for the $\mathrm{v}$ variant is not yet known, the phage SfX, which is responsible for the group 7;8 antigenic determinant, was inducible from the sequenced S. flexneri Xv strain 2002017 [5]. Therefore emergence and spread of novel S. flexneri serotypes in nature poses a significant public health threat globally and in particular in developing countries where S. flexneri is the predominant cause of shigellosis.

In order to reveal possible roles played by the serotype-converting phages in the emergence of new serotypes, and potential of emergence of novel serotypes through this mechanism in nature, we performed infection assays using SfI and SfX, the 2 most common serotype-converting bacteriophages carried by $S$. flexneri based on serotype frequency data $[5,19]$. We demonstrate that a novel serotype, named serotype $1 \mathrm{~d}$ was created in laboratory by infecting S. flexneri serotype X strains with a SfI phage or by sequential infection of serotype Y strain with SfX and SfI.

\section{Results and discussion}

\section{Creation of a new serotype, serotype $1 \mathrm{~d}$, through} serotype conversion with phages Sfl and SfX

Using the procedures described by Mavris et al. [12], 2 serotype-converting phages, SfI and SfX, were induced and isolated from S. flexneri serotype 1a strain 019 and serotype Xv strain 2002017 respectively. The 2 phages were then used to sequentially infect a serotype $Y$ strain 036 in different order.

We first performed sequential infection in the order of SfI and SfX. By infection with SfI, the S. flexneri serotype Y strain 036 was converted into serotype 1a (036_1a), which agglutinated with both diagnostic typing sera I and grouping sera 3;4 (also known as Y-5) as shown in Table 1. Strain 036_1a was then used for infection by SfX, but surprisingly, no plaques appeared, indicating the strain cannot be infected by SfX.

Next we performed infection in the order of SfX and SfI. The S. flexneri serotype Y strain 036 was converted to serotype $\mathrm{X}$ by phage SfX infection, which agglutinated only with serotype X-specific grouping sera 7;8. We named this strain as S. flexneri 036_X (Figure 1A and $1 \mathrm{~B}$ ). When 036_X was further infected with phage SfI, it was converted to a new serotype, which agglutinated with both of the diagnostic serotype 1a-specific typing sera I and serotype X-specific grouping sera 7;8, and were negative for all other type and group-specific sera (Figure 1A and 1B). The conventional serological identification results were further confirmed by Western-blot assay. As shown in Figure 1C, the lipopolysaccharide (LPS) pattern of the newly constructed strain was identical to that of serotype X strain 014 (Panel a, Figure 1C) and that of serotype 1a strain 019 (Panel c, Figure 1C), when probed by group specific sera 7;8 and type specific sera I respectively. Since the original serotype $\mathrm{Y}$ strain and its SfI convertant 1a strain can agglutinate with grouping sera $3 ; 4$, we also tested whether this antigen is detectable in serotype $1 \mathrm{~d}$. The LPS of the new serotype was not recognized by the grouping sera 3;4 (Panel b, Figure 1C). Additionally, serotype-specific genes, $g \operatorname{tr} X$ for phage SfX and gtrI for phage SfI, were detected from these new strains by PCR and sequencing of the PCR products.

S. flexneri serotype 1 has three known subtypes, 1a, 1b and $1 \mathrm{c}$, the agglutination patterns of which are defined

Table 1 Serological characterization of S. flexneri serotype $Y, X, 1 a, 1 b$ and $1 c$ using serotyping monoclonal antibodies (MASF)

\begin{tabular}{ccccccccccccc}
\hline Serotypes & \multicolumn{8}{c}{ Reaction with MASF } \\
\cline { 2 - 13 } & \multicolumn{1}{c}{ Type antigen specific } & \multicolumn{7}{c}{ Group antigen specific } & 1c \\
\cline { 2 - 12 } & I & II & IV-2 & V & VI & B & $3 ; 4 *$ & 6 & $7 ; 8$ & IV-1 \\
\hline Fy & - & - & - & - & - & + & + & - & - & - & - \\
\hline Fx & - & - & - & - & - & + & - & - & + & - & - \\
\hline F1a & + & - & - & - & - & + & + & - & - & - & - \\
\hline F1b & + & - & - & - & - & + & - & + & - & - & - \\
\hline F1c & - & - & - & - & - & + & - & - & - & - & + \\
\hline F1d & + & - & - & - & - & + & - & - & + & - & - \\
\hline
\end{tabular}

*Y-5 is a synonym of grouping 3;4 antisera 

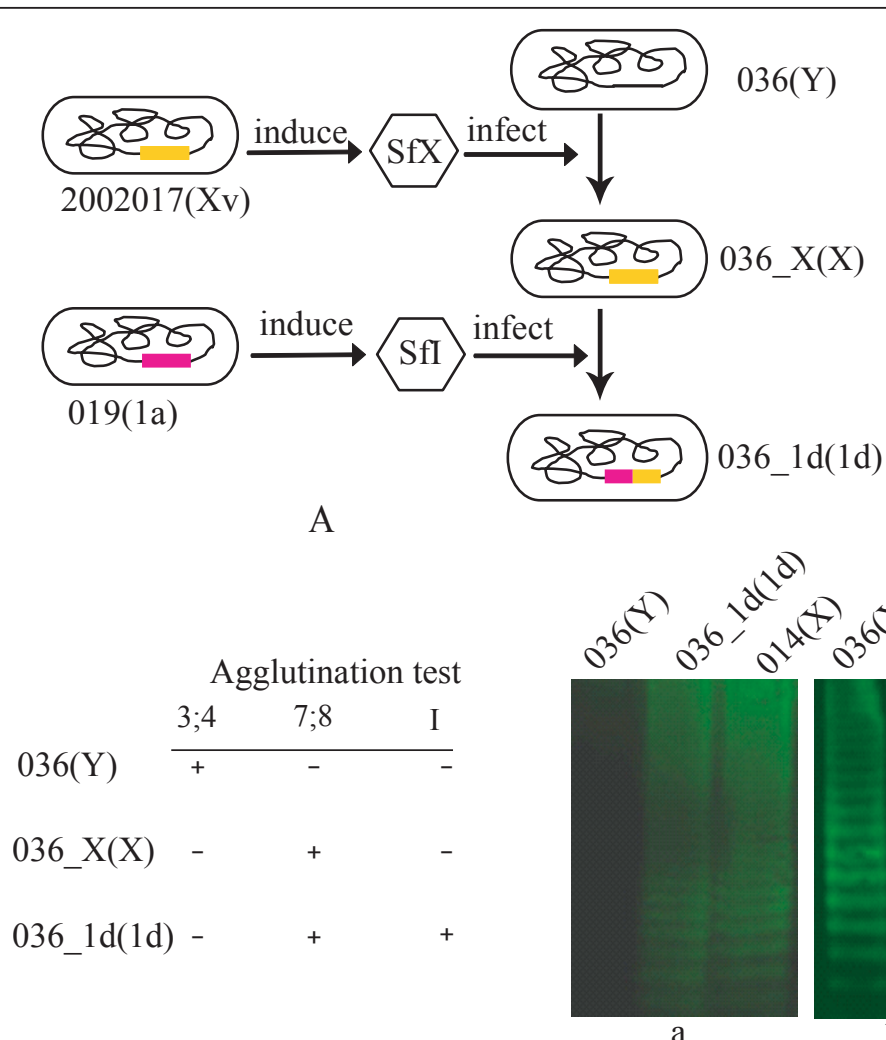

a

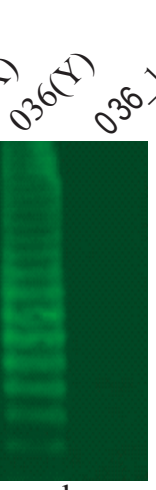

b

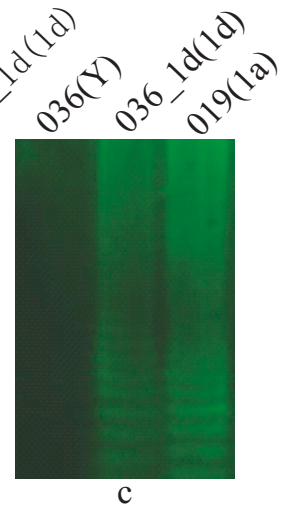

$\mathrm{C}$

Figure 1 Construction of a novel serotype, 1 d, of S. flexneri with serotype-converting bacteriophages SfX and Sfl. (A) Illustration of construction road map of S. flexneri 036_1d strain from a serotype $Y$ strain 036, by sequential infection of phages SfX and Sfl. (B) Serological identification of $\mathrm{S}$. flexneri 036_1d as serotype $1 \mathrm{~d}$ with agglutination test using monovalent diagnostic sera. The constructed strain S. flexneri 036_1d agglutinated with both of typing sera I and grouping sera7;8. (C) Serological identification of S. flexneri 036_1d by Western-blot assay. The LPS extracted from the tested strains was separated by SDS-PAGE and hybridized with monovalent grouping sera 7;8 (a) and 3;4 (b), and typing sera I (c), respectively. LPS of serotype X strain 014 and serotype 1a strain 019 were used as positive controls for group specific antigen 7;8 and type specific antigen I. After strain name in brackets is the serotype of the strain.

by a combination of typing and grouping sera, namely typing sera I and grouping sera 3;4 (Y-5) for 1a, typing sera I and grouping sera 6 for 1b, S. flexneri group antigen specific MASF $B$ and provisional specific monoclonal antibody MASF1c for 1c [17] (Table 1). Since the newly constructed serotype agglutinates with typing sera I, but showed a different serological pattern from all known serotype 1 subtypes (Table 1), we named this new serotype $1 \mathrm{~d}$.

In order to determine whether such serotype-converting events could occur in nature, we randomly selected 24. S. flexneri serotype $\mathrm{X}$ strains in our collection, and infected them with serotype-converting phage SfI. All 24 strains tested were successfully converted to serotype $1 \mathrm{~d}$.

We have no good explanation why serotype 1a strain 036_1a, constructed from 036 by infection with SfI, could not be further infected by SfX. We randomly selected 17 S. flexneri 1a isolates from our collection for infection by SfX but found that none of them could be infected by SfX. Clearly, the SfI can infect the strains carrying serotype-converting phage SfX, but not vice versa, likely due to phage immunity from modified Oantigen receptors [20].

Interestingly, a recent study reported $S$. flexneri strains with identical serological characteristics to the novel serotype $1 \mathrm{~d}$ created in this study [21]. Four strains were designated as untypeable serotype I: $(7 ; 8)$ among $467 \mathrm{~S}$. flexneri isolates collected in a passive surveillance project from Henan, China in 2006 [21]. Thus it seems that this novel serotype has already appeared in natural infections. Although serotype $1 \mathrm{~d}$ represented less than $1 \%$ of the isolates, it would be important to monitor this new serotype epidemiologically, considering that novel S. flexneri serotypes such as $1 \mathrm{c}$ and $\mathrm{Xv}$ achieved its dominance among the $S$. flexneri serotypes in a very short time frame $[5,16,17]$ 


\section{Sfl and SfX integrated in tandem into the same site of host chromosome}

It has been observed that the serotype-converting phages, except for Sf6, usually integrate into the $t R N A$ thrW gene of the host chromosome, which is adjacent to proA upstream [15]. However, the gene downstream the integrated phage have not been consistently identified [6,7]. Genomic analysis of S. flexneri serotype 2a strain 301 (NC_004337), 2457 T (NC_004741) and serotype Xv strain 2002017 (CP001383) showed that the serotype-converting phages were all integrated upstream of host gene yaiC. Thus cross-bridging PCR analyses of $S$. flexneri 036, 036_X, and 036_1d across the proA-yaiC region were conducted using a series of primers and found that both phages SfX and SfI were integrated into the $t R N A-t h r W$ site, which is immediately downstream of gene proA, and upstream of gene yaiC (Figure 2). The phage SfI was found to be integrated immediately upstream of SfX genome, with an att site at both ends (Figure 2). By comparing the joining sequences between the serotype-converting phage genomes, we found that the phage SfI was integrated at the attL site of phage SfX (see Additional file 1). The integration site for the 24 serotype $\mathrm{X}$ isolates converted by SfI was also found to be the same site and thus it appears that the integration is very site specific.

\section{Conclusions}

A novel serotype $1 \mathrm{~d}$ was constructed by sequentially infecting a serotype Y strain of S. flexneri with phage SfX and SfI, or by infecting clinical serotype $\mathrm{X}$ isolates with SfI. These results indicate that serotype conversion with phages SfI and SfX could occur in nature. However, the observation that the order of infection by the 2 phages affects convertibility of a strain indicates that serotype conversion is not only determined by the modification specific genes but also constrained by the properties of the serotype-converting phages. Our findings provide possible mechanisms how new serotypes of S. flexneri could emerge in nature.

\section{Methods}

\section{Bacterial strains and phages}

S. flexneri strains and serotype-converting bacteriophages used in this study were listed in Table 2. S. flexneri strain 036 (serotype Y) was used as host for phage infection and large propagation. S. flexneri strains 014 (serotype X) and 019 (serotype 1a) were used as positive controls in the serological assays for group specific antigen 7;8 and type specific antigen I respectively. Twenty four S. flexneri serotype X and 17 of S. flexneri serotype 1a strains isolated from patients and stored at National Institute for Communicable Disease Control and Prevention, China CDC (ICDC) were used for infection with serotype-converting phages SfI and SfX respectively.

Serotype-converting bacteriophages SfI and SfX were induced from S. flexneri serotype 1a strain 019 and serotype Xv strain 2002017 respectively, following the methods described by Mavris et al. [12].

\section{Phage infection and lysogen isolation}

We used the procedures described for lambda phage $(\Phi \lambda)$ for phage infection [22]. S. flexneri cells were inoculated into LB broth and incubated for $3 \mathrm{~h}$ at $37^{\circ} \mathrm{C}$ with aeration. Cells were harvested by centrifugation at $4000 \mathrm{rpm}$ and the cell density was adjusted to $2.0 \mathrm{OD}$ $\left(\mathrm{A}_{595} \mathrm{~nm}\right)$ with $\mathrm{MgSO}_{4}$ buffer $(10 \mathrm{mmol} / \mathrm{L})$. A proportion of cells $(200 \mu \mathrm{l})$ were infected with purified phages with phage to bacterial cell ratio of about 1:1000 and incubated for $20 \mathrm{~min}$ at $37^{\circ} \mathrm{C}$. The infected cells were mixed with $3 \mathrm{ml}$ semisolid agar (Luria Broth (LB) with 0.7\% agar) and immediately spread on LB solid agar plates. After incubation at $37^{\circ} \mathrm{C}$ for $20 \mathrm{~h}$, the lysogens were detected from turbid single colonies.

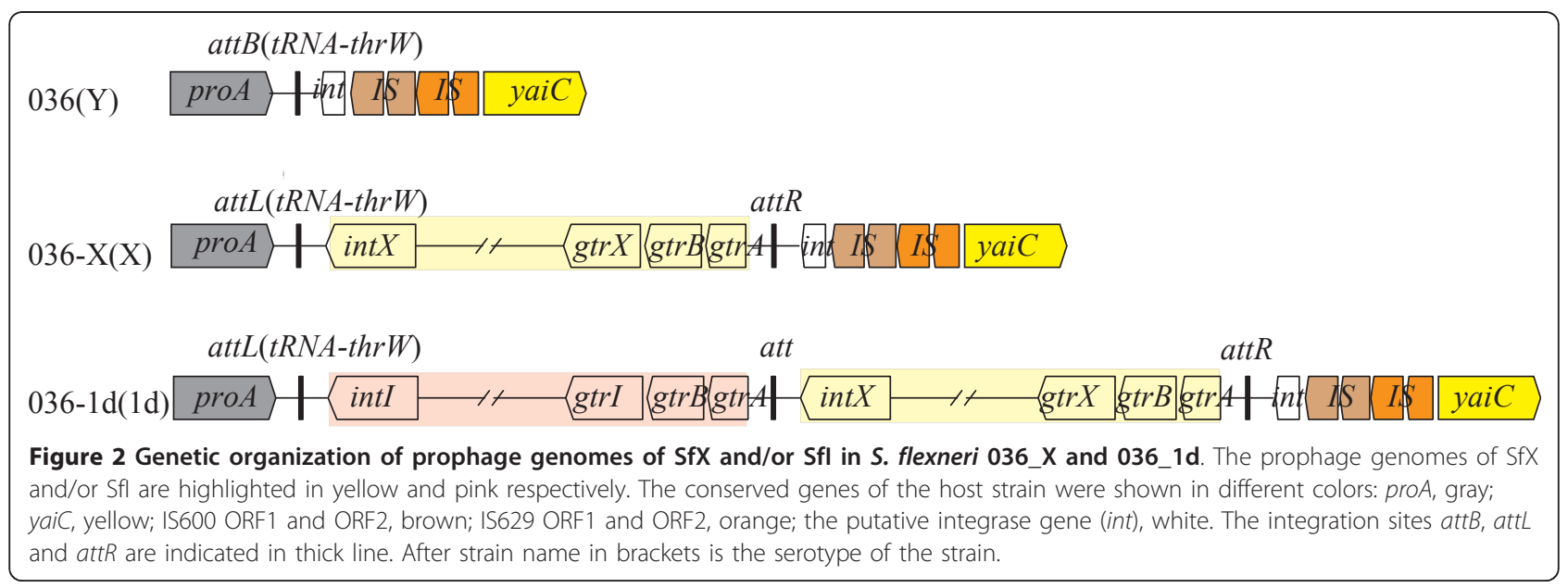


Table 2 Strains and serotype-converting bacteriophages used in this study

\begin{tabular}{lll}
\hline Strains or phages & Relevant characteristic & Reference or source \\
\hline S. flexneri strains & & ICDC \\
\hline 036 & Serotype $Y$ & ICDC \\
\hline 014 & Serotype $X$ & ICDC \\
\hline 019 & Serotype 1a & This study \\
\hline $036 \_1 a$ & 036 infected by Sfl, serotype 1a $X$ & This study \\
\hline $036 \_1 d$ & 036 infected by SfX, serotype $X$ & This study \\
\hline Phages & 036 infected by Sfl and SfX, serotype 1d & This study \\
\hline Sfl & & This study \\
\hline SfX & Phage Sfl, induced from S. flexneri strain 019 & \\
\hline
\end{tabular}

ICDC, National Institute for Communicable Disease Control and Prevention, China CDC

\section{Slide agglutination and LPS analysis}

Serological identification was performed using two commercial slide agglutination serotyping kits: monovalent anti-sera (Denka Seiken, Japan) and monoclonal antibody reagents (Reagensia $\mathrm{AB}$, Sweden) according to manufacturer's instructions. The new serotype was further confirmed by Western-blot assay. Briefly, LPS was prepared using the method of Hitchcock \& Brown [23] and transferred onto a PVDF membrane in a Tris/ glycine/methanol buffer. The membrane was blocked with phosphate buffered saline (PBS) containing 5\% (w/ v) skimmed milk and $0.05 \%$ Tween-20 and then incubated with serotype $\mathrm{X}$-specific monovalent grouping sera 7;8 (Denka Seiken, Japan), serotype 1a-specific monovalent typing sera I (Denka Seiken, Japan), or monovalent grouping sera 3;4 (Denka Seiken, Japan). The membrane was then washed with $1 \times$ PBS containing $0.05 \%$ Tween-20, and incubated with a secondary anti-rabbit antibody labeled with the fluorescent IRDye ${ }^{\text {TM }} 800$ (Rockland). Fluorescence was detected using an Odyssey Infrared Imaging System (LI-COR).

\section{Identification of the integration site and orientation of SfX and Sfl}

Based on previous studies showing that the integration site of serotyping-conversion bacteriophages is conserved [15], a series of primers were designed that were located in genes proA, yaiC, gtrI, gtrX, intI and int $X$ across the presumptive integration region to determine the site and order of integration using PCR: proA-F, ACAAAGCGAAATCATCCTCAA; intI-R, AGTGTTACAGGAAATGGGAGGC. $g t r I-F$, ATTGAACGCCTCCTTGCTATGC; int X-R, TACGGTGGCTGCGTGAGAA. $g \operatorname{tr} X-\mathrm{F}$, TACCTTGACCCGTTTCCG; and yaiC-R, GCAGGAAACCACCATCAACACC. PCR products were sequenced commercially to identify the integration site precisely.

\section{Additional material}

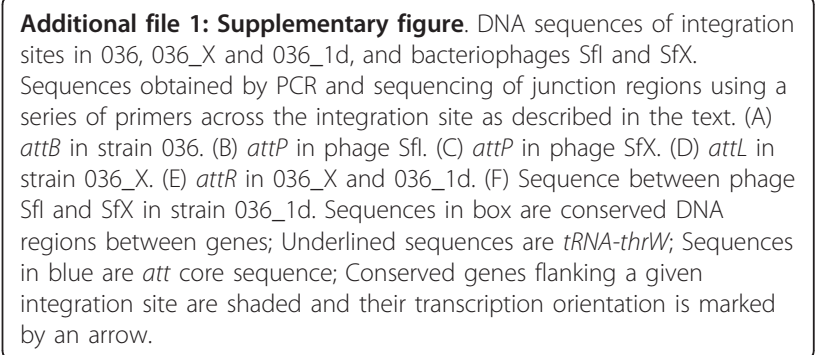

\section{Acknowledgements}

This work was supported by grants (2011CB504901, 2008ZX10004-008, 2008ZX10004-009, 2008ZX10004-001, 2009ZX10004-203, 2011SKLID203, 2008SKLID106, and YB20098450101) from the Ministry of Science and Technology, and State Key Laboratory for Infectious Disease Prevention and Control, People's Republic of China. We thank the referees for helpful suggestions.

\section{Author details}

${ }^{1}$ State Key Laboratory for Infectious Disease Prevention and Control, National Institute for Communicable Disease Control and Prevention, China CDC, P.O. Box 5, Changping, Beijing, China. ${ }^{2}$ School of Biotechnology and Biomolecular Sciences, University of New South Wales, Sydney, NSW 2052, Australia.

\section{Authors' contributions}

JX and QS designed the study, and co-drafted the manuscript. RL participated in the design of the study and preparation of the manuscript. YW participated in the construction of the new serotype. JW carried out the PCR amplification and DNA sequencing. XL performed the LPS Western-blot assay. SZ carried out the serological identification. PL participated in the phage induction and infection. $\mathrm{CY}$ and $\mathrm{HJ}$ participated in the isolation of clinical strains. YW participated in the sequence alignment. All authors read and approved the final manuscript.

Received: 16 July 2011 Accepted: 30 December 2011 Published: 30 December 2011

\section{References}

1. Kotloff KL, Winickoff JP, Ivanoff B, Clemens JD, Swerdlow DL, Sansonetti PJ, Adak GK, Levine MM: Global burden of Shigella infections: implications for vaccine development and implementation of control strategies. Bull World Health Organ 1999, 77(8):651-666. 
2. Bardhan P, Faruque AS, Naheed A, Sack DA: Decrease in shigellosis-related deaths without Shigella spp. -specific interventions, Asia. Emerg Infect Dis 2010, 16(11):1718-1723.

3. Bennish ML, Wojtyniak BJ: Mortality due to shigellosis: community and hospital data. Rev Infect Dis 1991, 13:S245-251.

4. Clemens JD, Kotloff KL, Kay B: Generic protocol to estimate the burden o Shigell diarrhoea and dysenteric mortalit. Geneva: World Health Organization; 1999.

5. Ye C, Lan R, Xia S, Zhang J, Sun Q, Zhang S, Jing H, Wang L, Li Z, Zhou Z, et al: Emergence of a new multidrug-resistant serotype $\times$ variant in an epidemic clone of Shigella flexneri. J Clin Microbiol 2010, 48(2):419-426.

6. Simmons DA, Romanowska E: Structure and biology of Shigella flexneri O antigens. J Med Microbiol 1987, 23(4):289-302.

7. Petrovskaya VG, Licheva TA: A provisional chromosome map of Shigella and the regions related to pathogenicity. Acta Microbiol Acad Sci Hung 1982, 29(1):41-53.

8. Clark CA, Beltrame J, Manning PA: The oac gene encoding a lipopolysaccharide O-antigen acetylase maps adjacent to the integraseencoding gene on the genome of Shigella flexneri bacteriophage Sf6. Gene 1991, 107(1):43-52.

9. Guan S, Bastin DA, Verma NK: Functional analysis of the $O$ antigen glucosylation gene cluster of Shigella flexneri bacteriophage SfX. Microbiology 1999, 145:1263-1273.

10. Allison GE, Angeles D, Tran-Dinh N, Verma NK: Complete genomic sequence of $\mathrm{SfV}$, a serotype-converting temperate bacteriophage of Shigella flexneri. J Bacteriol 2002, 184(7):1974-1987.

11. Casjens S, Winn-Stapley DA, Gilcrease EB, Morona R, Kuhlewein C, Chua JE, Manning PA, Inwood W, Clark AJ: The chromosome of Shigella flexneri bacteriophage Sf6: complete nucleotide sequence, genetic mosaicism, and DNA packaging. J Mol Biol 2004, 339(2):379-394.

12. Mavris M, Manning PA, Morona R: Mechanism of bacteriophage Sfllmediated serotype conversion in Shigella flexneri. Mol Microbiol 1997, 26(5):939-950.

13. Verma NK, Brandt JM, Verma DJ, Lindberg AA: Molecular characterization of the O-acetyl transferase gene of converting bacteriophage SF6 that adds group antigen 6 to Shigella flexneri. Mol Microbiol 1991, 5(1):71-75.

14. Huan PT, Bastin DA, Whittle BL, Lindberg AA, Verma NK: Molecular characterization of the genes involved in O-antigen modification, attachment, integration and excision in Shigella flexneri bacteriophage SfV. Gene 1997, 195(2):217-227.

15. Allison GE, Verma NK: Serotype-converting bacteriophages and O-antigen modification in Shigella flexneri. Trends Microbiol 2000, 8(1):17-23.

16. Stagg RM, Cam PD, Verma NK: Identification of newly recognized serotype $1 \mathrm{c}$ as the most prevalent Shigella flexneri serotype in northern rural Vietnam. Epidemiol Infect 2008, 136(8):1134-1140.

17. Talukder KA, Islam Z, Islam MA, Dutta DK, Safa A, Ansaruzzaman M, Faruque AS, Shahed SN, Nair GB, Sack DA: Phenotypic and genotypic characterization of provisional serotype Shigella flexneri 1c and clonal relationships with 1a and 1b strains isolated in Bangladesh. J Clin Microbiol 2003, 41(1):110-117.

18. Stagg RM, Tang SS, Carlin NI, Talukder KA, Cam PD, Verma NK: A novel glucosyltransferase involved in O-antigen modification of Shigella flexneri serotype 1c. J Bacteriol 2009, 191(21):6612-6617.

19. von Seidlein L, Kim DR, Ali M, Lee H, Wang X, Thiem VD, Canh do G, Chaicumpa W, Agtini MD, Hossain A, et al: A multicentre study of Shigella diarrhoea in six Asian countries: disease burden, clinical manifestations, and microbiology. PLoS Med 2006, 3(9):e353.

20. Bacterial surface polysaccharides and phage adsorption. Edited by: Lindberg AA. New York: Academic Press; 1977:

21. Xia S, Xu B, Huang L, Zhao JY, Ran L, Zhang J, Chen H, Pulsrikarn C, Pornruangwong S, Aarestrup FM, et al: Prevalence and characterization of human Shigella infections in Henan Province, China, in 2006. J Clin Microbiol 2011, 49(1):232-242.

22. Sambrook J, Fritsch EF, Maniatis T: Molecular Cloning: a Laboratory Manual. Cold Spring Harbor, NY: Cold Spring Harbor Laboratory; 21989.

23. Hitchcock PJ, Brown TM: Morphological heterogeneity among Salmonella lipopolysaccharide chemotypes in silver-stained polyacrylamide gels. J Bacteriol 1983, 154(1):269-277. doi:10.1186/1471-2180-11-269

Cite this article as: Sun et al:: Genesis of a novel Shigella flexneri serotype by sequential infection of serotype-converting bacteriophages SfX and Sfl. BMC Microbiology 2011 11:269.

\section{Submit your next manuscript to BioMed Central and take full advantage of:}

- Convenient online submission

- Thorough peer review

- No space constraints or color figure charges

- Immediate publication on acceptance

- Inclusion in PubMed, CAS, Scopus and Google Scholar

- Research which is freely available for redistribution

Submit your manuscript at www.biomedcentral.com/submit
Biomed Central 